\title{
Shannon entropy associated with electrochemically generated ion concentration gradients
}

\author{
N. V. Ryzhkov, V. Yu. Yurova, E. V. Skorb* \\ ITMO University, Lomonosova, 9, St. Petersburg, 191002, Russia \\ *skorb@itmo.ru
}

PACS 82.35.Rs, 82.39.Wj, 82.45.Fk, 82.60.Lf, 89.70.+c

DOI 10.17586/2220-8054-2020-11-2-171-175

\begin{abstract}
In this work, we discuss Shannon entropy in relation to ion distribution in solutions. Shannon entropy is a key concept of information theory. Discussion of ion solutions informational entropy is essential for consideration of ions as information carriers in iontronic devices. We studied entropy associated with ions redistribution using model electrochemically triggered local ion fluxes. For this purpose, we utilized bare gold electrodes as well as covered by polyelectrolyte layers and lipids. Modification of the electrode surface leads to a change of ion flux triggered by hydroquinone oxidation. Consequently, various distribution of ions in solution can be obtained. Shannon entropy was evaluated for diverse ion distributions.
\end{abstract}

Keywords: Shannon entropy, proton fluxes, layer by layer assemblies, electrochemistry.

Received: 30 December 2019

Revised: 17 February 2020

\section{Introduction}

Entropy is a function that quantifies the number of microstates available to a system. Thus, thermodynamic entropy growth is a driving force for many physicochemical processes. In solutions, the number of microstates at high concentrations is less than for low concentrations. For charged species, the electrostatic driving force opposes the entropic one.

Local ion signals can easily be generated using the electrochemical approach. Spatial propagation of protons produced during hydroquinone oxidation is supposed to be a three-dimensional $\mathrm{pH}$ wave described by Fick's laws [1]. The driving force for ion movement is the dispersal of energy into a more probable distribution. The quinone/hydroquinone redox process is characterized by relatively low redox potential, and both compounds are chemically stable. Due to this, quinones are widely used for electrochemically controlled generation and consumption of protons [2-4].

The diffusion of oxidation products is highly affected by the structure of the electrode surface $[5,6]$. Polyelectrolyte modified electrodes are of high interest [7,8]. Layer-by-layer (LbL) polyelectrolyte assemblies containing many charged moieties do not allow ions freely pass through. Polyelectrolyte protonation/deprotonation phenomenon is involved in ion pathways. A wide variety of applications are offered for LbL-assembled coatings and capsules. The LbL approach provides a versatile instrument for multilayer formation with the incorporation of variously charged compounds $[8,9]$. Polyelectrolyte multilayers are widely used in the design of biomaterial surface coatings [10,11], membranes for separation, as well as cargoes for drug encapsulation and delivery [12].

Thus, polyelectrolyte LbL assemblies can be considered as a convenient tool for regulation of ion signal transduction. Polyelectrolyte layers in multi-layered structures are not perfectly stratified, and polymer chains of polycations and polyanions are significantly interpenetrated [13]. Much attention is attracted to the dynamics and internal structure of polyelectrolyte multilayers and the phenomenon of internal diffusion $[14,15]$. Various parameters, such as ionic strength [16], charge density [17], $\mathrm{pH}$, and temperature [18], affect polyelectrolyte film internal structure, and several diffusion models in ultrathin polyelectrolyte films are described [19,20]. Except for permeability and nonpermeability, ions accumulation mode was also identified. Polyelectrolyte membrane permeability depends not only on the thickness, but also on film composition [21]. Multilayers composed solely of weak polyelectrolytes tend to transport small molecules or ions more preferably than ones assembled from strong polyelectrolytes [22]. Ion flux through polyelectrolyte membrane is also significantly affected by its stoichiometry, and excess of some component (polycation, or polyanion) affects ion permeability significantly [23]. Thus, the spatial and temporal distribution of electrochemically generated ionic fluxes can be easily tuned by polyelectrolyte membrane assembled from molecules with different molecular weights, strength, specific functional moieties.

Signal generation, storing and transmission via spatial and temporal ion distribution is a basis of iontronics, an emerging technology utilizing precise ion manipulation for information processing. An essential concept of information theory is Shannon entropy, which is a measure of the uncertainty amount. This study is devoted to establishing the relationship between ionic concentration in solution and Shannon entropy. Protons and hydroxide ions seem to 
be promising for the implementation of a chemical computer. These complementary particles neutralize each other when they are present simultaneously. Thus, it is possible to introduce a subtraction operation atypical for 'chemical arithmetic' [24]. Shannon entropy of solutions with various proton and hydroxide content is evaluated here.

\section{Materials}

Branched PEI (Mw $70 \mathrm{kDa}) 30 \%$ water solution purchased from Alfa Aesar; poly(sodium 4-styrene sulfonate) (PSS, Mw $500 \mathrm{kDa}$ ) purchased from Polysciences Inc.; liquid soya lecithin Lecisoy 400 containing mostly phosphatidylcholines obtained from Cargill, USA; reagent grade $\mathrm{NaCl}(99.5 \%)$ obtained from Merck and hexane from Ekos-1, Russia. All chemicals were used as received without any additional purification. Gold wire, $0.2 \mathrm{~mm}$ in diameter, was purchased from Agar (UK). Pieces of gold wire of $2-3 \mathrm{~cm}$ length were embedded in epoxy resin so that circular cross-section of gold wire exposed to outside media on the flat surface of the obtained holder (Fig. 1a). The deposition of the polyelectrolyte cushion onto the gold microelectrode embedded in the epoxy resin was performed using the classical LbL technique. $2 \mathrm{mg} / \mathrm{ml}$ each PEI and PSS was dissolved in $0.5 \mathrm{M}$ aqueous $\mathrm{NaCl}$ to make polycation and polyanion solutions, respectively. A total of seven layers were deposited, resulting in a $[\mathrm{PEI} / \mathrm{PSS}]_{3} / \mathrm{PEI}$ polyelectrolyte multilayer. Lecithin vesicles were obtained according to classical rehydration technique [25]. And lipid bilayer deposited from vesicles suspension [25] forming a continuous bilayer.

\section{Ion concentration measurements}

Proton flux was triggered at the solid-liquid interface by the oxidation of $60 \mathrm{mM}$ hydroquinone solutions in $150 \mathrm{mM} \mathrm{KNO}_{3}$ at a constant electric current of $5 \mathrm{~mA}$ at gold microelectrode embedded in epoxy resin. The concentration of protons close to the surface of gold microelectrode was investigated using Scanning Ion-Selective Electrode Technique (SIET). This instrumentation is based on potentiometric principles which allows characterizing local ionic currents in solution and ion concentration gradient measurement [26] (Fig. 1b). A glass capillary microelectrode filled by an ion-selective membrane in the tip scans the surface, measuring extremely low concentrations of a particular ion with micrometer spatial resolution (Fig. 1c). A system from Applicable Electronics (USA) modulated by an ASET program (Sciencewares, USA) was used to perform SIET measurements. SIET pH measurements were carried out using glass capillary microelectrodes filled with Hydrogen Ionophore Cocktail I (Sigma) and $\mathrm{KCl}+\mathrm{KH}_{2} \mathrm{PO}_{4}$ internal solution. $\mathrm{Ag} / \mathrm{AgCl} / \mathrm{KCl}$ (sat) was used as the external reference electrode.

a)

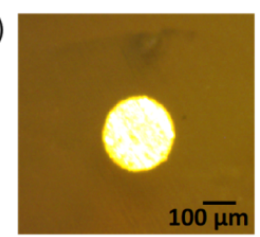

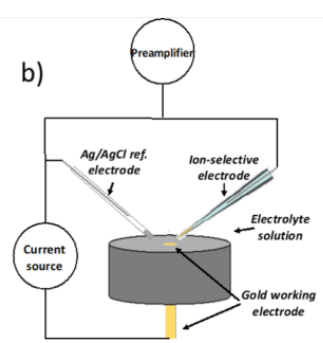

c)

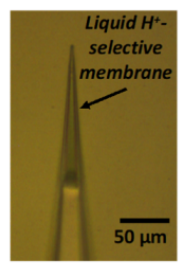

FIG. 1. a) Top view of gold electrode embedded in epoxy resin, b) Scheme of SIET measurement,

c) microphotography of glass capillary proton-sensitive electrode

The $\mathrm{pH}$-selective microelectrodes were calibrated using commercially available $\mathrm{pH}$ buffers. The local activity of $\mathrm{H}^{+}$was detected $25 \mathrm{~mm}$ above the surface and mapped on an $11 \times 11$ grid.

\section{Results and discussion}

Electron transfer in the quinone-hydroquinone system is associated with proton transfer. When hydroquinone is oxidized electrochemically, acidification of the near-electrode region occurs. A two-step hydroquinone oxidation was performed at a gold microelectrode surface (Fig. 2a).

Cyclic voltammetry curves were not affected by the further deposition of polyelectrolyte layers and lipid layer formation (Fig. 2a). Since molecules of hydroquinone and quinone are rather small and uncharged, they can pass freely through polyelectrolyte/lipid layer, and multilayer assembly on top of electrodes leads to no change in redox processes at the electrode/hydroquinone solution interface.

Zwitterionic lipids can adsorb protons; thus, at neutral conditions, phosphate groups exist in a deprotonated state. As some components of soy lecithin, in this case, carry an only negative charge, which is not compensated by the positive amine group (e.g., phosphatidylinositol and phosphatidic acid) [27], there is a net negative charge for the lipid 

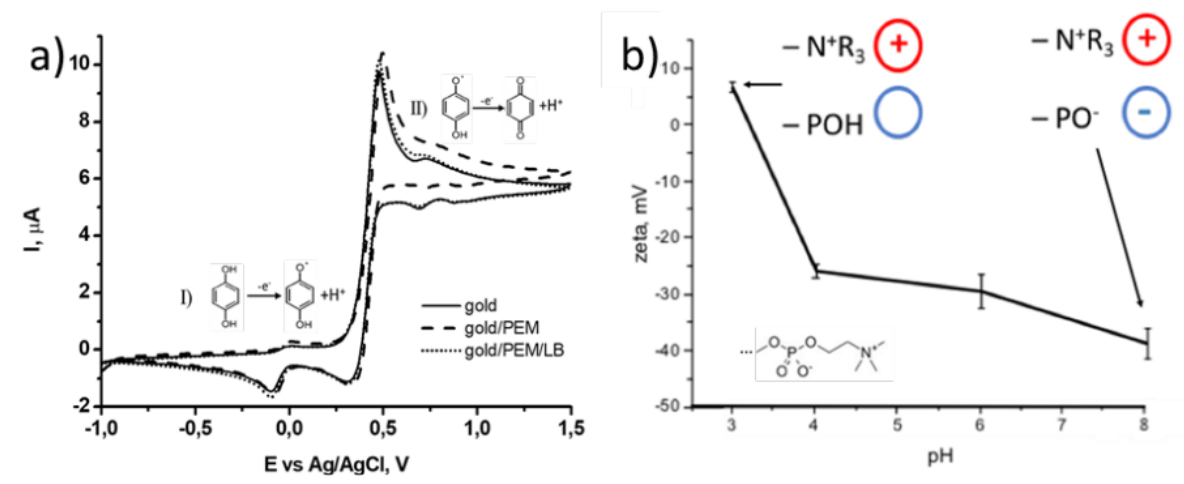

FIG. 2. a) Cyclic voltammetry curves of hydroquinone on a pure gold electrode and a gold electrode covered by a polyelectrolyte multilayer and polymer cushioned lipid layer, b) $\zeta$-potential of lipid vesicles obtained from soy lecithin at different $\mathrm{pH}$ adjusted by diluted $\mathrm{HCl}$ and $\mathrm{NaOH}$ solutions

structures. In acidic media, due to protonated phosphate groups, a positive net charge occurs. The pH-dependence lipid's charge was demonstrated by vesicles $\zeta$-potential measurements (Fig. 2b).

SIET measured ion concentration and obtained $\mathrm{pH}$ maps were recalculated to entropy maps for pristine gold working electrode (WE) (Fig. 3a), WE covered with polyelectrolyte multilayers gold/(PEI/PSS) 3 /PEI (Fig. 3b), and WE covered with polyelectrolyte multilayers and lipid layer on top gold/(PEI/PSS) 3 /PEI/lipid (Fig. 3c).

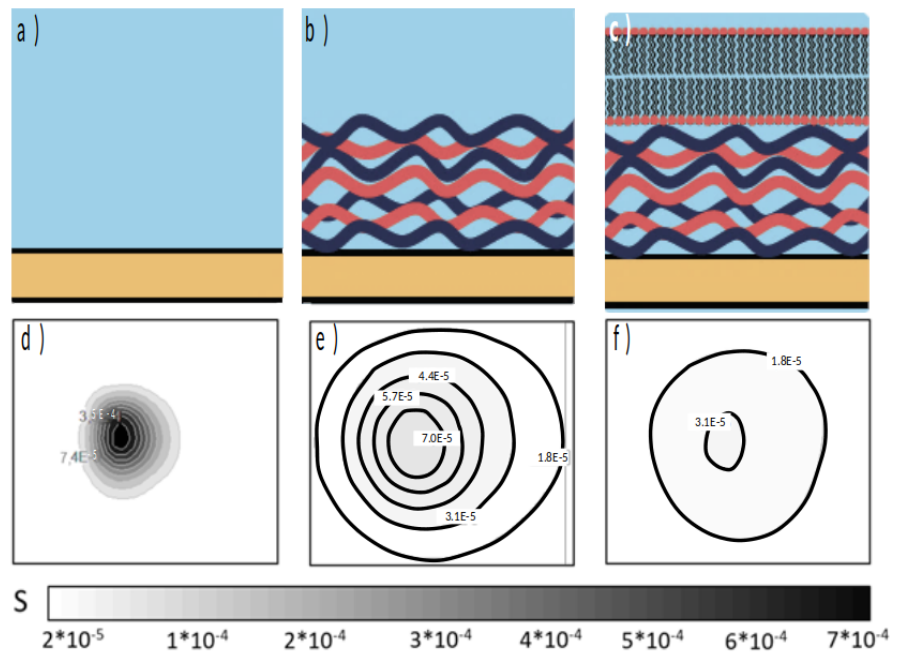

FIG. 3. a) Pristine gold electrode, b) gold electrode covered by polyelectrolyte multilayer gold/(PEI/PSS $\left.)_{3} / \mathrm{PEI}, \mathrm{c}\right)$ polyelectrolyte modified gold electrode with lipid bilayer deposited on top gold/(PEI/PSS $)_{3} / \mathrm{PEI} / \mathrm{LB}, \mathrm{d}-\mathrm{f}$ ) recalculation of $\mathrm{pH}$ maps registered by SIET into Shannon's entropy for (d) pristine gold working electrode, e) gold working electrode covered with polyelectrolytes gold/(PEI/PSS $)_{3} / \mathrm{PEI}$, and f) gold working electrode covered with polyelectrolytes and lipid bilayer gold/(PEI/PSS $)_{3} / \mathrm{PEI} / \mathrm{LB}$; process occurring at electrode is oxidation of hydroquinone associated with proton flux; lines connect points with equal measured entropy values

Shannon's entropy is a fundamental concept of information theory. This value is a measure of unpredictability of the state, which is equal to its average information content. Since ions are good candidates to perform chemical calculations, we consider electrochemically generated ion fluxes in terms of Shannon entropy, which is a crucial concept of information theory.

Self-organization processes are closely related to entropy change [6]. Entropy change usually relates to a change of thermodynamic parameters such as temperature and pressure or chemical parameters. In the case of electrochemically driven ion flux, the changes in temperature and pressure in the system are negligible, while ion-distribution in the solution is disturbed 
Spatial redistribution of protons takes place self-consistently and occurs together with a redistribution of local electric potential during the minimization of the system free-energy. Therefore, the self-organization and the redistribution of $\mathrm{pH}$-fields are directly associated with each other and, thus, the change of entropy can be illustrated using the fields of $\mathrm{pH}$.

Shannon's entropy is defined as:

$$
S=-\sum_{i=1}^{n} p_{i} \log _{2} p_{i}
$$

where $0 \leq p_{i} \leq 1$ is the probability of measuring some observable value $i$ [28].

For instance, $p$ can be related to the probability of some molecular dipole orientation or electric charge in case of electric systems, an electron spin orientation or magnetic polarization in magnetic systems, a particular state of photons in optical systems, or concentration of chemicals in reacting systems [29]. In the same manner, this approach could be naturally expanded to solutions, to consider $p$ in sense of probability that an observed ion in the solution is an $\mathrm{H}^{+}$or $\mathrm{OH}^{-}$ion (whose concentrations are related with each other). The physical sense of the probability $p$ depends on the particular system's nature. We are interested in a system of $\mathrm{H}, \mathrm{OH}$ and non-dissociated water only. In accordance with this, we will consider the Shannon entropy for this subsystem only. From the concentration of ions, we can calculate the probability $p_{H}$ and $p_{O H}$ of finding them at a certain point. In other words, we may use the parts of $\mathrm{H}^{+}$and $\mathrm{OH}^{-}$ ions as the probabilities $p_{H}$ and $p_{O H}$, to calculate corresponding contributions to the Shannon's entropy associated with the $\mathrm{pH}$ in a given spatial area of measurement. Hence, considering only the entropy subsystem containing $\mathrm{H}, \mathrm{OH}$ and water, Shannon's entropy associated with the measured $\mathrm{pH}$ in a particular spatial area can be calculated as follows:

$$
S=-\left(p_{H} \log _{2} p_{H}+p_{O H} \log _{2} p_{O H}+\left(1-p_{O H}-p_{H}\right) \log _{2}\left(1-p_{O H}-p_{H}\right)\right) .
$$

The last term takes into account the contribution provided by other molecules which we do not measure in experiment $\left(\mathrm{H}_{2} \mathrm{O}\right)$.

The probability of observing proton, hydroxide-ion or non-dissociated water in a solution is related to its concentration, i.e. $\mathrm{pH}$ as:

$$
p_{H}=C_{H} / C_{0}=10^{-p H},
$$

where $C_{0}=1 M$ is a normalizing concentration. For $\mathrm{OH}^{-}$anions, we have:

$$
p_{O H}=C_{O H} / C_{0}=10^{-p O H}=10^{p H-14},
$$

since $p H+p O H=14$ in water solutions and $p_{H_{2} O}=1-p_{H}-p_{O H}$. From here, by substitution of these expressions for $p$ into the equation (2), we obtain Shannon's entropy contribution associated with $p_{H}$ measured in a small volume:

$$
S=-\left(10^{-p H} \log _{2} 10^{-p H}+10^{p H-14} \log _{2} 10^{p H-14}+\left(1-10^{p H-14}-10^{-p H}\right) \log _{2}\left(1-10^{p H-14}-10^{-p H}\right)\right) \text {. }
$$

The first two terms here are associated with the $\mathrm{H}$ and $\mathrm{OH}$ subsystems, whereas the last one is due to the (subsystem of) non-dissociated water molecules. In vicinity of the neutral $\mathrm{pH}$, the last term provides about $6 \%$, while the main contribution is provided by:

$$
S \approx-\left(10^{-p H} \log _{2} 10^{-p H}+10^{p H-14} \log _{2} 10^{p H-14}\right),
$$

that we used for the illustration of the experiments in Fig. 3, without losses of the conclusion generality related to the engineering of a given $\mathrm{pH}$ distribution with polyelectrolytes.

Usually, as a substance becomes more dissipated, the thermal energy carried by this substance also spreads over volume, followed by entropy. Meanwhile, Shannon entropy associated with $\mathrm{pH}$ being dependent on complementary proton and hydroxide-ion concentration is not a monotonous function. The function described by the equation above exhibits a local minimum at $\mathrm{pH}=7$. The summation of Shannon entropy values can estimate overall entropy for the particular spatial distribution of protons (i.e., distribution of the system states) overall points of the system.

The approach based on the equation above is convenient for analysis of self-organization phenomena (related to change in pH-distributions) and their interpretation in terms of entropy fields. This can be illustrated using the results of our measurements of $\mathrm{pH}$ fields in different systems.

Considering the system described above, we can easily vary ion distribution. For instance, taking the spatial distribution of cations determined experimentally in cases of a bare electrode, as well as for electrode covered by polyelectrolyte multilayer (PEI/PSS) 3 and lipid bilayer, we obtained the spatial distributions of entropy $S(x, y)$ represented in Figs. 3(a-c). Interestingly, a huge difference is observed for obtained the spatial distributions of entropy that can be associated with various soft matter components for controlling ion fluxes. 


\section{Conclusion}

A model electrochemical system creating local ion-fluxes was demonstrated. The LbL assembly of polyelectrolyte multilayers is suggested as an instrument to control horizontal and vertical ion propagation with the ability to correlate it with the spatial distributions of entropy. In contradistinction to thermodynamic entropy, informational Shannon entropy associated with decreased ionic redistribution with solution dilution. The spatial distribution of Shannon entropy was studied for ion fluxes generated electrochemically.

\section{Acknowledgements}

This work is supported by RSF Grant No. 17-79-20186. EVS also thanks the ITMO Fellowship Professorship Program for Infrastructural Support. The authors thank Prof. Stanislav O. Yurchenko (BMSTU, Moscow) for the discussion of entropy analysis.

\section{References}

[1] Fomina N., Johnson C.A., et al. An electrochemical platform for localized pH control on demand. Lab Chip., 2016, 16, P. $2236-2244$.

[2] Garnier T., Dochter A., et al. Surface confined self-assembly of polyampholytes generated from charge-shifting polymers. Chem. Commun., 2015, 51, P. 14092-14095.

[3] Dochter A., Garnier T., et al. Film Self-Assembly of Oppositely Charged Macromolecules Triggered by Electrochemistry through a Morphogenic Approach. Langmuir, 2015, 31 (37), P. 10208-10214.

[4] Ryzhkov N.V., Mamchik N.A., Skorb E.V. Electrochemical triggering of lipid bilayer lift-off oscillation at the electrode interface. J. R. Soc. Interface, 2019, 16 (150), 20180626.

[5] Maza E., Tuninetti J.S., et al. pH-responsive ion transport in polyelectrolyte multilayers of poly(diallyldimethylammonium chloride) (PDADMAC) and poly(4-styrenesulfonic acid-co-maleic acid) (PSS-MA) bearing strong- and weak anionic groups. Phys. Chem. Chem. Phys., 2015, 17, P. 29935-29948.

[6] Ryzhkov N.V., Nesterov P., et al. Localization of ion concentration gradients for logic operation. Front. Chem., 2019,7 , 419.

[7] Ryzhkov N.V., Andreeva D.V., Skorb E.V. Coupling pH-Regulated Multilayers with Inorganic Surfaces for Bionic Devices and Infochemistry. Langmuir, 2019, 35 (26), P. 8543-8556.

[8] Ryzhkov N.V., Brezhneva N., Skorb E.V. Feedback mechanisms at inorganic polyelectrolyte interfaces for applied materials. Surf. Innov., 2019, 7 (3-4), P. 145-167.

[9] Decher G., Eckle M., Schmitt J., Struth B. Layer-by-layer assembled multicomposite films. Curr. Opin. Colloid Interface Sci., 1998, 3 (1), P. 32-39.

[10] Zhukova Y., Hiepen C., et al. The Role of Titanium Surface Nanostructuring on Preosteoblast Morphology, Adhesion, and Migration. Adv. Healthc. Mater., 2017, 6 (15), 1601244.

[11] Rmaile H.H., Farhat T.R., Schlenoff J.B. pH-gated Permeability of Variably Charged Species through Polyelectrolyte Multilayer Membranes. J. Phys. Chem. B, 2003, 107 (51), P. 14401-14406.

[12] Nikitina A.A., Ulasevich S.A., et al. Nanostructured Layer-by-Layer Polyelectrolyte Containers to Switch Biofilm Fluorescence. Bioconjug. Chem., 2018, 29 (11), P. 3793-3799.

[13] Schönhoff M. Self-assembled polyelectrolyte multilayers. Curr. Opin Colloid Interface Sci., 2003,8 (1), P. 86-95.

[14] Selin V., Ankner J.F., Sukhishvili S.A. Nonlinear Layer-by-Layer Films: Effects of Chain Diffusivity on Film Structure and Swelling. Macromolecules, 2017, 50 (16), P. 6192-6201.

[15] Fares H.M., Schlenoff J.B. Diffusion of Sites versus Polymers in Polyelectrolyte Complexes and Multilayers. J. Am. Chem. Soc., 2017, 139 (41), P. 14656-14667.

[16] Steitz R., Leiner V., Siebrecht R., Klitzing R. Influence of the ionic strength on the structure of polyelectrolyte films at the solid/liquid interface. Colloids Surfaces A Physicochem. Eng. Asp., 2000, 163 (1), P. 63-70.

[17] Steitz R., Jaeger W., Klitzing R. Influence of charge density and ionic strength on the multilayer formation of strong polyelectrolytes. Langmuir, 2001, 18 (4), P. 1408-1412.

[18] Karg M., Pastoriza-Santos I., et al. Temperature, pH, and ionic strength induced changes of the swelling behavior of PNIPAM-poly(allylacetic acid) copolymer microgels. Langmuir, 2008, 24 (12), P. 6300-6306.

[19] Klitzing R., Möhwald H. A realistic diffusion model for ultrathin polyelectrolyte films. Macromolecules, 1996, 29 (21), P. $6901-6906$.

[20] Farhat T.R., Schlenoff J.B. Ion transport and equilibria in polyelectrolyte multilayers. Langmuir, 2001,17 (4), P. 1184-1192.

[21] Hoshi T., Saiki H., Anzai J.I. Amperometric uric acid sensors based on polyelectrolyte multilayer films. Talanta, 2003 , 61 (3), P. $363-368$.

[22] Fu J., Fares H.M., Schlenoff J.B. Ion-Pairing Strength in Polyelectrolyte Complexes. Macromolecules, 2017,50 (3), P. $1066-1074$.

[23] Kelly K.D., Fares H.M., Abou Shaheen S., Schlenoff J.B. Intrinsic Properties of Polyelectrolyte Multilayer Membranes: Erasing the Memory of the Interface. Langmuir, 2018, 34 (13), P. 3874-3883.

[24] Banda P., Teuscher C., Stefanovic D. Training an asymmetric signal perceptron through reinforcement in an artificial chemistry. J. R. Soc. Interface, 2014, 11 (93), 20131100.

[25] Diamanti E., Gregurec D., et al. Lipid layers on polyelectrolyte multilayers: Understanding lipid-polyelectrolyte interactions and applications on the surface engineering of nanomaterials. J. Nanosci. Nanotechnol., 2016, 16 (6), P. 5696-5700.

[26] Souto R.M., Lamaka S.V., González S. Uses of Scanning Electrochemical Microscopy in Corrosion Research. In: Microscopy: Science, Technology, Applications and Education, 2010.

[27] Scholfield C.R. Composition of Soybean Lecithin. J. Am. Oil Chem. Soc., 1981, 58 (10), P. 889-892.

[28] Shannon C.E. A Mathematical Theory of Communication. Bell Labs Tech. J., 1948, 27 (3), P. $379-423$.

[29] Haken H. Information and Self-Organization. A Macroscopic Approach to Complex Systems. Springer, Berlin, 2006,257 p. 